\title{
DIVERSIDADE ALFA E BETA NO CERRADO SENSU STRICTO DA CHAPADA PRATINHA, BRASIL.
}

\author{
Maria Cristina Felfili ${ }^{1}$ \\ Jeanine Maria Felfili
}

Recebido em 27/07/99. Aceito em 02/05/01

\begin{abstract}
RESUMO - (Diversidade alfa e beta no cerrado sensu stricto) O bioma cerrado possui uma das mais ricas floras dentre as savanas mundiais com mais de 6000 espécies, abrange uma vasta extensão territorial, contém as três maiores bacias hidrográficas sul americanas, e se destaca pela elevada biodiversidade. O objetivo deste trabalho, que faz parte do Projeto Biogeografia do Bioma Cerrado, foi analisar as diversidades alfa e beta em comunidades de cerrado sensu stricto. Foram amostrados o Parque Nacional de Brasília, a Estação Ecológica de Águas Emendadas, a Area de Proteção Ambiental (APA) Gama-Cabeça de Veado, Silvânia-GO, Paracatu-MG e Patrocínio-MG. A amostragem foi aleatória com 10 parcelas de 20x50m em cada área de estudo. Foram incluídas plantas lenhosas, exceto lianas, que tivessem no mínimo $5 \mathrm{~cm}$ de diâmetro. Foi calculada a diversidade alfa pelo teste de Shannon \& Wienner e o de Simpson. A diversidade beta foi calculada pelo índice de Whittaker que mede a mudança ou taxa de substituição na composição de espécies de um local para outro. Foi efetuada a curva espécie-área para as 60 parcelas amostradas e efetuada a classificação por TWINSPAN. O cerrado sensu stricto da Chapada Pratinha pode ser diferenciado em duas zonas fitogeográficas: Distrito Federal-Silvânia e Paracatu-Patrocínio. Estas coincidem com um zoneamento por sistemas de terra que classifica a primeira zona como terras altas em contraposição à segunda.
\end{abstract}

Palavras-chave - Cerrado, diversidade alfa e beta, Chapada Pratinha-DF.

\begin{abstract}
Alfa and Beta diversity in the cerrado sensu stricto) The cerrado flora is one of the richest among the world's savannas with more than 6000 species. The cerrado covers a large territory, contains the three most important hydrographycal basins in South America and a high diversity. The objective of this work, that is part of the project Biogeography of the Cerrado Biome, was to analyse alfa and beta diversity in the cerrado sensu stricto. The sampled places were: Brasília National Park - DF, Águas Emendadas Ecological Station - DF, Environmentally protected Area of Gama-Cabeça de Veado - DF, Silvânia - GO, Paracatu - MG and Patrocínio - MG. The sampling was randomized with 10 plots of $20 x 50 \mathrm{~m}$ in each study-site. Woody plants from $5 \mathrm{~cm}$ dbh, excluding lianas were sampled. Shannon \& Wienner's and Simpson's diversity index were used to calculate alfa diversity and Whittaker's index for beta diversity. The latter measures the rate of replacement in species composition from one place to another. A species-area curve was also drawn for the sixty plots sampled. A classification by the TWINSPAN method was also carried out. The cerrado sensu stricto in Chapada Pratinha can be separated in two phytogeographic zones: Distrito Federal - Silvânia and Paracatu - Patrocínio. Those coincide with a land system classification which places the first zone in the highlands and the second in the lowlands.
\end{abstract}

Key words - Cerrado, savanna, alfa diversity, beta diversity, Brazil.

\footnotetext{
${ }^{1}$ Instituto Brasileiro do Meio Ambiente - IBAMA

${ }^{2}$ Departamento de Engenharia Florestal Universidade de brasília CP 04357, 70919970 Brasília-DF.
} 


\section{Introdução}

O cerrado sensu stricto (s.s.), que ocupa aproximadamente $70 \%$ do Bioma Cerrado tem paisagem composta por um estrato herbáceo dominado principalmente por gramíneas e, um estrato de árvores e arbustos variando em cobertura de 10 a $60 \%$ (Eiten 1972). A vegetação do Cerrado ocorre sobre vários tipos de solo, mas a maior parte destes (c. 46\%) são solos bem drenados, profundos, ácidos, pobres em nutrientes e com alta saturação de alumínio (Adámoli et al. 1987).

Vários estudos descreveram os principais tipos físionômicos e composição florística da vegetação do Cerrado (Eiten 1972, 1984; Goodland 1971; Ratter et al. 1978; Ratter 1991), que se apresenta distribuída em gradientes (Coutinho 1978), com variação apreciável na fisionomia, florística, abundância e diversidade de espécies (Goodland 1971; Goodland \& Ferri 1979; Ribeiro et al. 1981; Oliveira Filho et al. 1989; Felfili \& Silva Júnior 1993; Felfili et al. 1994, 1997). Em decorrência das diferenças de solo e relevo, espera-se a ocorrência de diferentes comunidades. Os trabalhos que têm analisado este problema ainda são poucos (Ratter \& Dargie 1992; Felfili \& Silva Junior 1993; Ratter et al. 1996).

A flora do cerrado está entre as mais ricas dentre as savanas do mundo, com uma estimativa superior a 6.000 espécies vasculares (Mendonça et al. 1998) e já foi reconhecido internacionalmente como um dos 25 hot spots para conservação (Mittermayer et al. 1999) em função da sua elevada diversidade biológica sob ameaça pela ocupação desordenada que já converteu mais de $50 \%$ da vegetação natural em paisagens antropizadas (Klink et al. 1995; Klink 1996; Mittermayer et al. 1999). Considerando que as espécies de plantas têm uma distribuição restrita (Ratter \& Dargie 1992; Felfili et al. 1994; Ratter et al. 1996; Felfili et al.
1997), a magnitude da devastação da flora do cerrado pode ser muito grande.

As pesquisas sobre o modo como está organizada e distribuída a biodiversidade nas comunidades do cerrado, são ainda reduzidas. Estas informações são necessárias para avaliar os impactos decorrentes de atividades antrópicas, planejar a criação de unidades de conservação e para a adoção de técnicas de manejo.

Em ambientes tropicais o número de espécies é elevado e a distribuição desigual com poucas espécies contendo muitos indivíduos e um grande número de espécies com um número reduzido de indivíduos, podendo ser consideradas raras localmente. No cerrado sensu stricto, a diversidade alfa, está na faixa de 3,0 a 3,5 nats/indivíduo para amostras de um hectare compostas por parcelas disjuntas (Felfili et al. 1994; 1997) sendo portanto, elevada (Magurran 1988). A diversidade beta entre locais, expressa por índices de similaridade é, em geral, elevada quando são utilizados índices que levam em conta presença e ausência de espécie mas decresce quando são utilizados índices quantitativos podendo atingir valores baixos. Até o momento, os estudos comparativos foram feitos entre locais, ou seja, fez-se uma amostragem em certa localidade, encontrou-se os valores médios e comparou-se com resultados de outras localidades obtidos de modo similar (Felfili et al. 1994; 1997). Análises multivariadas têm revelado um padrão de mosaico para a diversidade do cerrado (Ratter \& Dargie 1992; Felfili \& Silva Júnior 1993; Felfili et al. 1997).

O objetivo deste trabalho foi analisar a diversidade de espécies em várias comunidades de cerrado (sensu stricto), comparando parcelas dentro de uma mesma localidade e entre localidades da Chapada Pratinha, procurando elucidar quais seriam as diversidades alfa e beta nas comunidades. Procurou-se também, aprofundar os resultados encontrados por Felfili et al. (1994) analisando os padrões de 
distribuição das comunidades de cerrado da Chapada Pratinha ao nível local, investigando se as comunidades de cerrado mais próximas, são as mais similares. Procurou-se também, associar os padrões encontrados com o zoneamento do cerrado efetuado por Cochrane et al. (1985) que tem servido de base para o projeto Biogeografia do Bioma Cerrado.

\section{Material e métodos}

\section{Área do estudo}

A chapada Pratinha (Felfili et al.1994), está situada nos estados de Minas Gerais, Goiás e Distrito Federal, com seus limites entre as latitudes $15^{\circ}$ e $20^{\circ} \mathrm{S}$ e longitudes $46^{\circ}$ e $49^{\circ} \mathrm{W}$. A altitude média da chapada está em torno de 1000 metros. Os solos são bem drenados, a precipitação média anual é de 1600 $\mathrm{mm}$ e a temperatura média anual é de $20^{\circ} \mathrm{C}$. A forma predominante do relevo é de planalto, cortado por vales com declividade média inferior a $30 \%$. Esta unidade fisiográfica (Cochrane et al. 1985) contém dois sistemas de terra: Terras Altas e Terras Baixas da Chapada Pratinha cujas características físicas e fisionômicas estão descritas conforme o citado zoneamento e a situação quanto a ações antrópicas foram observações pessoais das autoras.

\section{Descrição das Áreas Amostrais}

Foram selecionadas seis áreas de estudo. As quatro primeiras áreas de estudo situam-se nas Terras Altas da Chapada Pratinha enquanto que as duas últimas estão nas baixas conforme o zoneamento elaborado por Cochrane et al. (1985). Estas foram:

1 - Parque Nacional de Brasília no Distrito Federal (DF), (PNB/DF), com área de 30.000 ha, localizado entre as coordenadas $15^{\circ} 37^{\prime}$ a $15^{\circ} 45^{\prime} \mathrm{S}$ e 47 $54^{\prime}$ ' a $47^{\circ} 59^{\prime} \mathrm{W}$, com altitude média de 1100 metros, tendo o cerrado sensu stricto (s.s.) como fitofisionomia predominante.
2 - Área Proteção Ambiental Gama-Cabeça de Veado (APA)/DF: Compreende as unidades de conservação da Fazenda Água Limpa da Universidade de Brasília, Reserva Ecológica do IBGE e Jardim Botânico de Brasília DF. A APA tem 25.000 ha, e as unidades de conservação nela contidas somam uma área de cerca de 9.000 ha; localiza-se entre as coordenadas $15^{\circ} 52^{\prime}$ a $15^{\circ} 59^{\prime} \mathrm{S}$ e $47^{\circ} 50^{\prime}$ a $47^{\circ} 58^{\prime} \mathrm{W}$, com altitude média de 1.100 metros. Apresenta vegetação com predomínio da fisionomia cerrado s.s., contendo também, extensas área de campo sujo e campo limpo.

3 - Estação Ecológica de Água Emendadas (EEAE)/DF: Área de 10.000 ha, localiza-se entre as coordenadas $15^{\circ} 31^{\prime}$ a $15^{\circ} 35^{\prime} \mathrm{S}$ e $47^{\circ} 32^{\prime}$ a $47^{\circ} 37^{\prime} \mathrm{W}$, com altitude média em torno de $1.100 \mathrm{~m}$. A vegetação predominante é o cerrado s.s. ocorrendo também extensas áreas cobertas por veredas.

4 - Silvânia/GO

Nesta área de estudo a amostragem foi realizada entre as coordenadas $16^{\circ} 30^{\prime}$ a $16^{\circ} 50^{\prime} \mathrm{S}$ e $48^{\circ} 30^{\prime}$ a $48^{\circ} 46^{\prime} \mathrm{W}$, incluindo parte dos municípios de Leopoldo de Bulhões, Silvânia e Vianópolis. A altitude média desta região está em torno de $1050 \mathrm{~m}$. Extensos plantios de soja estão sendo implantados na região, assim como muitas áreas com pastagens que vêm ocupando o lugar dos cerrados. A vegetação predominante é o cerrado s.s. porém, com extensas áreas de cerradão. 5 - Paracatu/MG

A área amostrada situou-se entre $17^{\circ} 00^{\prime}$ a $17^{\circ} 20^{\prime} \mathrm{S}$ e $46^{\circ} 45^{\prime}$ a $47^{\circ} 07^{\prime} \mathrm{W}$ com altitude média de $900 \mathrm{~m}$, vegetação de cerrado s.s. e outras fisionomias, bastante fragmentadas por intensa atividade agrícola.

6 - Patrocínio/MG

A amostragens foi realizada entre $18^{\circ} 47^{\prime}$ a $19^{\circ} 45^{\prime} \mathrm{S}$ e $46^{\circ} 25^{\prime}$ a $47^{\circ} 09^{\prime} \mathrm{W}$, incluindo áreas dos municípos de Patrocínio, Ibiá e Pratinha, com altitude média de $950 \mathrm{~m}$. Dentre os locais amostrados, foi onde se notou 
maior ação antrópica, com grandes áreas cultivadas, principalmente com café e soja. Esta foi a área de maior extensão, devido principalmente à dificuldade de se encontrarem áreas amostrais com vegetação não perturbada. $\mathrm{O}$ cerradão predomina no município de Patrocínio e o cerrado s.s. nos demais.

\section{Amostragem da Vegetação}

Foram utilizadas 10 parcelas de 20 x $50 \mathrm{~m}$ $\left(1000 \mathrm{~m}^{2}\right)$ em cada um dos seis locais estudados. Utilizou-se das cartas do IBGE na escala 1: 100.000 para delimitar as áreas amostrais. Após excursão exploratória para verificação em campo, selecionou-se as áreas com vegetação natural remanescente sem fortes vestígios antrópicos, classificadas como não perturbadas. Procedeu-se a um sorteio para a alocação das parcelas (Philip 1994). Nestas parcelas foram medidos os diâmetros a $0,30 \mathrm{~m}$ do solo de todos os indivíduos que apresentaram um mínimo de $5 \mathrm{~cm}$. Todo o material coletado foi identificado por comparação no herbário da Reserva Ecológica do Instituto Brasileiro de Geografia e Estatística (IBGE), e duplicatas foram enviadas para especialistas de diversas instituições nacionais e estrangeiras para a completa identificação.

\section{Análise dos Dados}

Uma das unidades básicas em que está organizada a biodiversidade são as comunidades. Esta organização apresenta dois componentes: diversidade alfa e diversidade beta. A diversidade alfa refere-se ao número e a abundância de espécies dentro de uma comunidade, enquanto que a diversidade beta, se relaciona com as diferenças na composição de espécies e suas abundâncias entre áreas dentro de uma comunidade (Margurran 1988).

A magnitude da diversidade alfa está relacionada com a riqueza ou número de espécies por unidade de área e a equabilidade ou seja a distribuição do número de indivíduos por espécie.
Para avaliar a diversidade alfa nas comunidades foram utilizados os índices de Shannon-Wienner (H'), que atribui maior peso a espécies raras, e o índice de Simpson (D), que é pouco influenciado por espécies raras (Magurran 1988).

A diversidade beta foi avaliada pelo índice de Whittaker (b), que mede a mudança ou taxa de substituição na composição de espécies de um local para outro (Whittaker 1960). Este índice varia de 0, quando duas amostras não apresentam nenhuma diferença na composição de espécies e 2, quando esta diferença é máxima, sendo calculado pela fórmula: $\beta=(\mathrm{c} / \mathrm{a})-1$, onde: $\mathrm{c}=$ total de espécies nas parcelas amostradas; $a=$ média do número de espécies das parcelas amostradas.

Para cada área foi calculada a diversidade beta entre todos os pares de parcelas, calculando-se o valor médio e o desvio padrão para cada uma das seis áreas.

Uma curva de incremento do número de espécies por parcela foi preparada para cada local. O incremento foi calculado seqüencialmente para cada uma das parcelas de cada local. Foi também elaborada outra curva para as 60 parcelas amostradas para verificar o incremento ao nível regional. As parcelas foram ordenadas conforme gradientes latitudinais e altitudinais sugeridos como os principais determinantes na vegetação da Chapada (Felfili \& Silva Júnior 1993; Felfili et al. 1997).

Uma matriz contendo 137 espécies por 60 parcelas tendo a densidade como variável foi utilizada para a classificação pelo método TWINSPAN ("Two-way species indicator analysis"). Neste método constrói-se uma tabela ordenada de dupla entrada. Primeiro as amostras são classificadas por dicotomização e depois as espécies pelo mesmo processo usando a classificação das amostras como base (Hill et al. 1975). Foi também construída, uma matriz de presença e ausência. 
Felfili \& Silva Júnior (1993) já fizeram a comparação entre locais considerando os valores médios dos conjuntos de 10 parcelas por local e a análise da composição florística está publicada em Felfili et al. (1994).

\section{Resultados e Discussão}

Em todas os locais amostrados, houve um número elevado de espécies (mais de um quarto) nos primeiros $1.000 \mathrm{~m}^{2}$ de cada área amostral, com aumento gradativo de espécies, até a última parcela (Fig. 1). A curva conjunta para todas as áreas, também mostrou um aumento gradativo até a parcela 30 (parcelas do DF na ordem amostrada), havendo em seguida um incremento paulatino de espécies até a parcela 50 (parcelas de Silvânia e Paracatu) a partir de onde se observa um acréscimo acentuado (parcelas de Patrocínio) (Fig. 2).

A distribuição de espécies por área mostrou-se semelhante a outra área de cerrado amostrada pelo mesmo método por Felfili \& Silva Júnior (1992) com um número elevado de espécies nas parcelas iniciais. O posterior aumento paulatino de espécies indica que a maioria das espécies ocorreram nas primeiras parcelas, sendo necessário um distanciamento elevado para que novas espécies surjam, ou seja, a diversidade beta entre parcelas próximas é baixa (indicando uma similaridade elevada entre parcelas próximas). Este padrão indica uma distribuição em mosaicos constatada por Felfili \& Silva Júnior (1993) onde um grupo de espécie domina sobre áreas de cerrado gerando alta similaridade entre locais, sendo necessário um elevado distanciamento ou diferenciações marcantes nas condições do terreno para que a similaridade entre áreas cobertas por esta vegetação seja reduzida. Esta distribuição é bastante diferente daquela encontrada para matas de galeria e matas amazônicas onde ocorrem menos espécies por parcelas de um mesmo tamanho mas o acréscimo a cada nova parcela é maior, ou seja a diferenciação entre locais próximos sobre a mesma condição ambiental é maior (Felfili 1995).

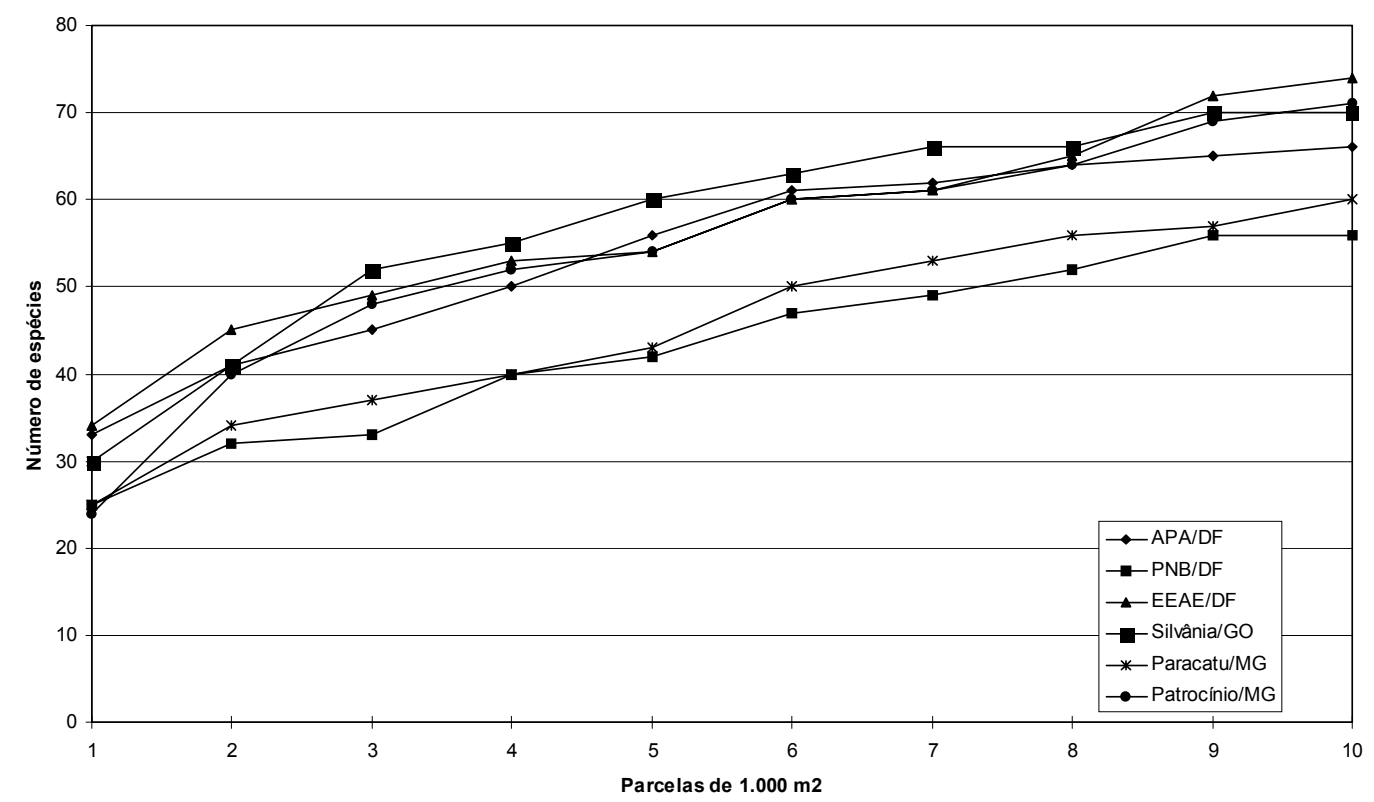

Figura 1 - Curva de incremento do número de espécie por parcelas amostradas para seis áreas de cerrado sensu stricto da Chapada Pratinha 


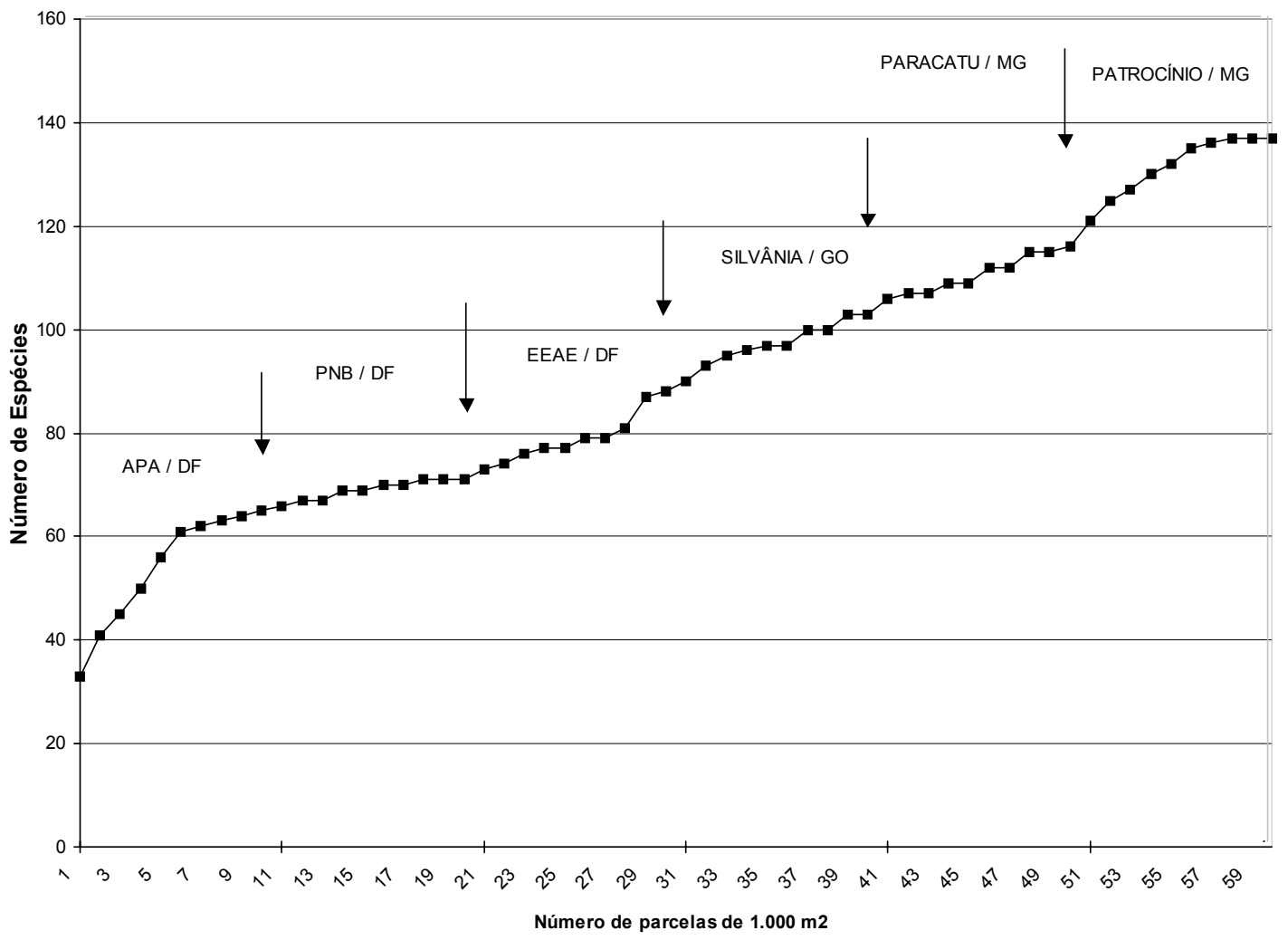

Figura 2-Curva de incremento do número de espécies por parcelas para o cerrado (APA/DF 1 - 10; PARNA/DF 11 - 20; EEAE DF 21 - 30; Silvânia/GO 31 - 40; Paracatu/MG 41 - 50 e Patrocínio/MG 51 - 60). As setas assinalam o final de cada área.

A APA Gama-Cabeça de Veado e a Estação Ecológica de Águas Emendadas foram as áreas mais ricas com o número de espécies por parcela da ordem de 30 a 45. As mais pobres foram Paracatu e Patrocínio com valores na ordem de 15 a 30 espécies por parcela (Tab. 1).

As parcelas do Distrito Federal e Silvânia apresentaram um nível similar de diversidade alfa, em torno de 3,0 pelo índice de Shannon e de 0,9 pelo de Simpson (Tab. 1). Esta decresce nas parcelas de Paracatu e Patrocínio, com o índice de Shannon em torno de 2,5 e algumas parcelas apresentando índice de Simpson em torno de 0,8. A diversidade beta (Fig. 3) foi menor na APA-Gama Cabeça de Veado $(0,30)$ no Distrito Federal e atingiu seu maior valor em Patrocínio-MG $(0,59)$ com as demais áreas atingindo valores intermediários. Ou seja, na APA, em Águas Emendadas e em Silvânia há uma maior homogeneidade no cerrado do que nas demais áreas. A diversidade beta entre parcelas de Paracatu e Patrocínio, apresentou grande variação, como pode ser constatado pelos desvios padrões elevados (Fig. 3).

Não existem outros estudos que tenham avaliado deste modo a diversidade beta nas comunidades dos cerrados, o que impossibilita as comparações com os resultados apresentados neste trabalho. As observações mostraram os seguintes padrões: (a) a diversidade beta varia geograficamente de até duas vezes o seu valor inicial; (b) a proporção em 
TABELA 1 - Diversidade Alfa no cerrado sensu stricto na Chapada Pratinha.

\begin{tabular}{|c|c|c|c|c|c|}
\hline & ÍNDICE & & ÍNDICE & & \\
\hline Parcela & Shannon-Wienner & Equabilidade & Simpson & Equabilidade & Número de espécies \\
\hline APA-Gama/DF 1 & 3.070 & 0.878 & 0.945 & 0.270 & 33 \\
\hline APA-Gama/DF 2 & 2.980 & 0.885 & 0.931 & 0.276 & 29 \\
\hline APA-Gama/DF 3 & 3.280 & 0.922 & 0.960 & 0.270 & 35 \\
\hline APA-Gama/DF 4 & 3.175 & 0.925 & 0.960 & 0.279 & 31 \\
\hline APA-Gama/DF 5 & 3.032 & 0.867 & 0.943 & 0.270 & 33 \\
\hline APA-Gama/DF 6 & 3.309 & 0.869 & 0.948 & 0.249 & 45 \\
\hline APA-Gama/DF 7 & 3.156 & 0.881 & 0.944 & 0.263 & 36 \\
\hline APA-Gama/DF 8 & 3.030 & 0.891 & 0.940 & 0.277 & 30 \\
\hline APA-Gama/DF 9 & 3.384 & 0.911 & 0.963 & 0.259 & 41 \\
\hline APA-Gama/DF 10 & 3.205 & 0.894 & 0.952 & 0.266 & 36 \\
\hline PNB/DF 11 & 2.801 & 0.881 & 0.926 & 0.291 & 24 \\
\hline $\mathrm{PNB} / \mathrm{DF} 12$ & 2.742 & 0.842 & 0.900 & 0.276 & 26 \\
\hline $\mathrm{PNB} / \mathrm{DF} 13$ & 2.039 & 0.850 & 0.858 & 0.358 & 11 \\
\hline $\mathrm{PNB} / \mathrm{DF} 14$ & 2.817 & 0.855 & 0.927 & 0.281 & 27 \\
\hline $\mathrm{PNB} / \mathrm{DF} 15$ & 2.749 & 0.918 & 0.933 & 0.311 & 20 \\
\hline $\mathrm{PNB} / \mathrm{DF} 16$ & 2.918 & 0.876 & 0.929 & 0.279 & 28 \\
\hline $\mathrm{PNB} / \mathrm{DF} 17$ & 3.029 & 0.891 & 0.944 & 0.277 & 30 \\
\hline $\mathrm{PNB} / \mathrm{DF} 18$ & 2.974 & 0.949 & 0.956 & 0.305 & 23 \\
\hline $\mathrm{PNB} / \mathrm{DF} 19$ & 2.942 & 0.926 & 0.947 & 0.298 & 24 \\
\hline PNB/DF 20 & 2.661 & 0.888 & 0.926 & 0.309 & 20 \\
\hline EEAE/DF 21 & 3.058 & 0.874 & 0.940 & 0.269 & 33 \\
\hline EEAE/DF22 & 3.109 & 0.914 & 0.952 & 0.280 & 30 \\
\hline EEAE/DF 23 & 3.138 & 0.906 & 0.952 & 0.275 & 32 \\
\hline EEAE/DF 24 & 3.102 & 0.859 & 0.935 & 0.259 & 37 \\
\hline EEAE/DF 25 & 2.886 & 0.886 & 0.937 & 0.287 & 26 \\
\hline EEAE/DF 26 & 3.252 & 0.915 & 0.958 & 0.269 & 35 \\
\hline EEAE/DF 27 & 2.992 & 0.863 & 0.937 & 0.270 & 32 \\
\hline EEAE/DF 28 & 2.937 & 0.820 & 0.919 & 0.257 & 36 \\
\hline EEAE/DF 29 & 3.119 & 0.877 & 0.946 & 0.266 & 35 \\
\hline EEAE/DF 30 & 2.975 & 0.884 & 0.938 & 0.279 & 29 \\
\hline Silvânia/GO 31 & 2.858 & 0.849 & 0.922 & 0.274 & 29 \\
\hline Silvânia/GO 32 & 2.738 & 0.813 & 0.908 & 0.270 & 29 \\
\hline Silvânia/GO 33 & 3.078 & 0.888 & 0.946 & 0.273 & 32 \\
\hline Silvânia/GO 34 & 2.608 & 0.810 & 0.878 & 0.273 & 25 \\
\hline Silvânia/GO 35 & 2.894 & 0.843 & 0.925 & 0.269 & 31 \\
\hline Silvânia/GO 36 & 2.633 & 0.829 & 0.911 & 0.287 & 24 \\
\hline Silvânia/GO 37 & 2.510 & 0.812 & 0.890 & 0.288 & 22 \\
\hline Silvânia/GO 38 & 2.761 & 0.858 & 0.924 & 0.287 & 25 \\
\hline Silvânia/GO 39 & 2.921 & 0.828 & 0.922 & 0.261 & 34 \\
\hline Silvânia/GO 40 & 2.727 & 0.780 & 0.891 & 0.255 & 33 \\
\hline Paracatu/MG 41 & 2.705 & 0.851 & 0.915 & 0.288 & 24 \\
\hline Paracatu/MG 42 & 2.569 & 0.788 & 0.874 & 0.268 & 26 \\
\hline Paracatu/MG 43 & 2.142 & 0.773 & 0.839 & 0.303 & 16 \\
\hline Paracatu/MG 44 & 2.095 & 0.756 & 0.808 & 0.291 & 16 \\
\hline
\end{tabular}




\begin{tabular}{llllll}
\hline & ÍNDICE & & ÍNDICE & \\
\hline Parcela & Shannon-Wienner & Equabilidade & Simpson & Equabilidade & Número de espécies \\
\hline Paracatu/MG 45 & 2.132 & 0.769 & 0.827 & 0.298 & 16 \\
Paracatu/MG 46 & 2.537 & 0.862 & 0.909 & 0.309 & 19 \\
Paracatu/MG 47 & 2.225 & 0.843 & 0.868 & 0.329 & 14 \\
Paracatu/MG 48 & 2.447 & 0.847 & 0.889 & 0.308 & 18 \\
Paracatu/MG 49 & 2.611 & 0.922 & 0.930 & 0.328 & 17 \\
Paracatu/MG 50 & 3.025 & 0.918 & 0.954 & 0.289 & 27 \\
Patrocínio/MG 51 & 3.205 & 0.996 & 0.997 & 0.310 & 27 \\
Patrocínio/MG 52 & 2.392 & 0.726 & 0.855 & 0.259 & 17 \\
Patrocínio/MG 53 & 2.267 & 0.800 & 0.850 & 0.300 & 22 \\
Patrocínio/MG 54 & 2.516 & 0.814 & 0.885 & 0.286 & 22 \\
Patrocínio/MG 55 & 2.873 & 0.929 & 0.943 & 0.305 & 18 \\
Patrocínio/MG 56 & 2.411 & 0.834 & 0.878 & 0.304 & 18 \\
Patrocínio/MG 57 & 2.444 & 0.846 & 0.896 & 0.310 & 23 \\
Patrocínio/MG 58 & 2.713 & 0.865 & 0.919 & 0.293 & 22 \\
Patrocínio/MG 59 & 2.626 & 0.850 & 0.898 & 0.291 & 17 \\
Patrocínio/MG 60 & 2.470 & 0.878 & 0.945 & 0.270 & \\
\hline
\end{tabular}

que a diversidade beta muda dentro da comunidade pode variar consideravelmente, sugerindo uma elevada heterogeneidade florística especialmente entre as parcelas de Paracatu-MG.

Áreas com valores altos de diversidade beta, ou seja, que apresentam elevada diferenciação entre parcelas próximas, requerem unidades de conservação de maior porte para abranger sua diversidade florística do que aquelas com baixa diversidade beta. $\mathrm{Na}$ Chapada Pratinha, as Terras baixas não são comtempladas com Unidades de Conservação, que estão todas concentradas no DF, que se localiza nas Terras Altas.

A variação geográfica nestes padrões pode ser o resultado de um padrão natural como também refletir diferentes graus de perturbação humana no entorno das áreas estudadas. Paracatu e Patrocínio são áreas de colonização antiga, já povoadas desde o ciclo da mineração, são zonas de pecuária, cafeicultura e outros cultivos agrícolas. Portanto o cerrado é fragmentado e sob forte pressão antrópica, contrastando com a região do DF onde este ocorre de maneira contínua dentro de Unidades de Conservação.

A primeira divisão da classificação por TWINSPAN, usando como parâmetro a densidade das espécies por ha, foi forte (autovalor de 0,318 ) e separou as parcelas do Distrito Federal e Silvânia da maioria das parcelas de Paracatu e de Patrocínio (Fig. 4). A ordem de classificação das parcelas do Distrito Federal e de Silvânia coincidiu com a seqüência de amostragem no campo, ou seja, parcelas próximas foram mais similares entre si. Estes resultados apoiam as sugestões de Ratter \& Dargie (1992) e Felfili \& Silva Junior (1993) de que o cerrado da Chapada Pratinha se distribui em mosaicos.

As espécies indicadoras do primeiro grupo foram Kielmeyera coriacea, Ouratea hexasperma e Styrax ferrugineus, todos com dois a cinco indivíduos por ha além de Dalbergia miscolobium e Stryphnodendron adstringens com 1 a 2 indivíduos por ha cada. Curatella americana com 5 a 10 indivíduos por ha foi a espécie indicadora do segundo grupo. A segunda divisão foi fraca (autovalor 


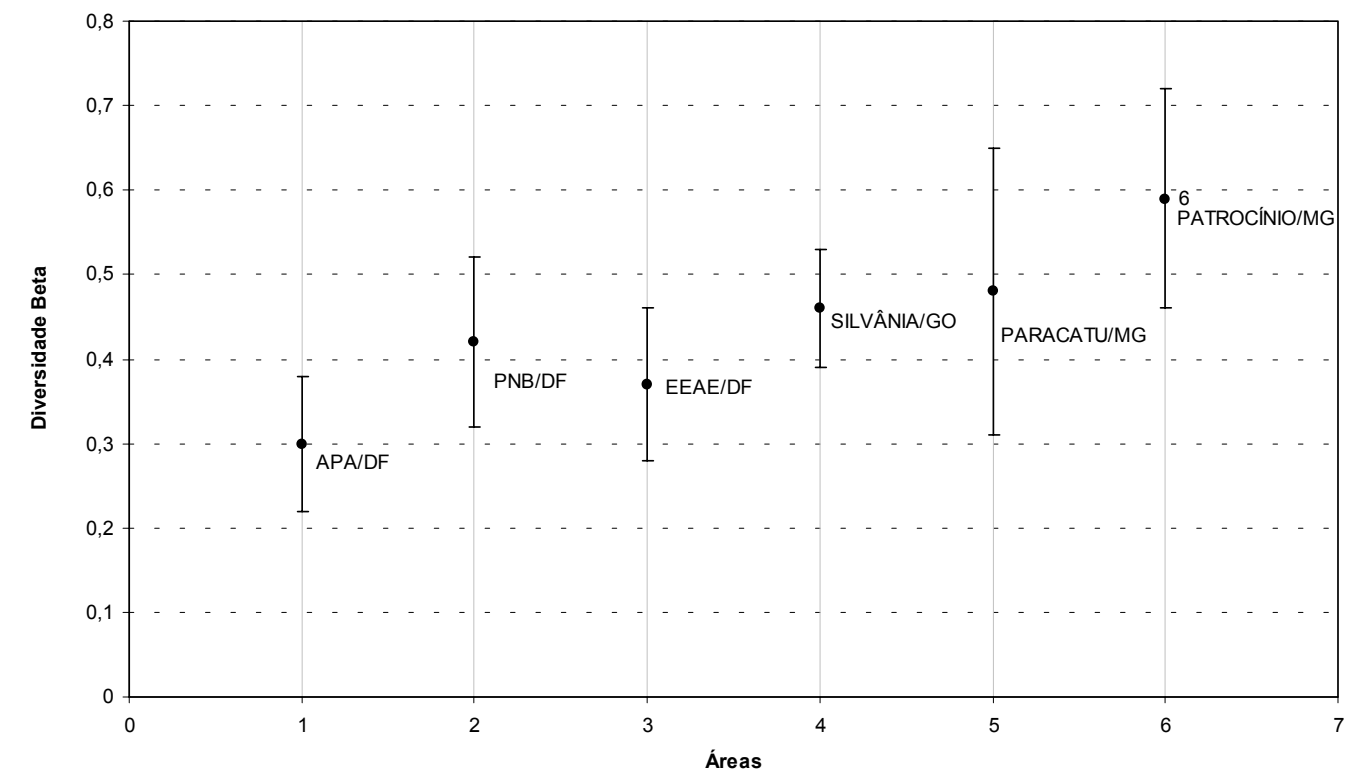

Figura 3 - Diversidade Beta (médias e desvios) no cerrado sunsu stricto da Chapada Pratinha. 1 - APA/DF; 2. PNB/DF; 3 EEAE/DF; 4 - Silvânia/GO; 5 - Paracatu/MG e 6 - Patrocínio/MG.

de 0,256). A terceira divisão, foi forte, com autovalor de 0,515 e separou as parcelas de Paracatu das de Patrocínio no segundo grupo. Acosmium dasycarpum com 1 a 2 indivíduos por ha foi a indicadora de Patrocínio.

As divisões geradas pela classificação de TWINSPAN usando a presença e ausência das espécies nas parcelas foram fracas (autovalor de 0,256 na primeira divisão).

Os agrupamentos foram similares e até mesmo as espécies indicadoras foram coincidentes. Estes resultados também reforçam as conclusôes de Felfili \& Silva Júnior (1993) e Felfili et al. (1997) na comparação entre locais, quando encontraram similaridade baixa para o índice de Sørensen que considera apenas presença e ausência de espécies.

A estrutura da comunidade parece ser o mais importante fator na diferenciação entre as localidades na Chapada Pratinha, haja visto, que apenas espécies de baixa densidade foram indicadoras da zona Distrito Federal e Silvânia e que a classificação baseada em presença e ausência de espécies não foi significante. Ou seja, do ponto de vista florístico, a Chapada é um contínuo se diferenciando em comunidades devido a diferenças na densidade de populações. Estes resultados reforçam as sugestões de Felfili et al. (1994) e Felfili \& Silva Júnior (1993).

As unidades de conservação da Chapada Pratinha são pesquenas, a maior é o Parque Nacional de Brasília e estão todas concentradas no Distrito Federal ficando a zona fitogeográfica Paracatu-Patrocínio desprotegida. A diferenciação na estrutura das populações entre as comunidades de cerrado nas duas zonas, evidenciada pela classificação por TWINSPAN com base na densidade das mesmas em cada comunidade, indica a necessidade de criação e consolidação de unidades de conservação nas terras baixas da Chapada Pratinha.

\section{Conclusões}

O cerrado sensu stricto da Chapada Pratinha pode ser diferenciado em duas zonas fitogeográficas, Distrito Federal-Silvânia e Paracatu-Patrocínio, especialmente em fun- 


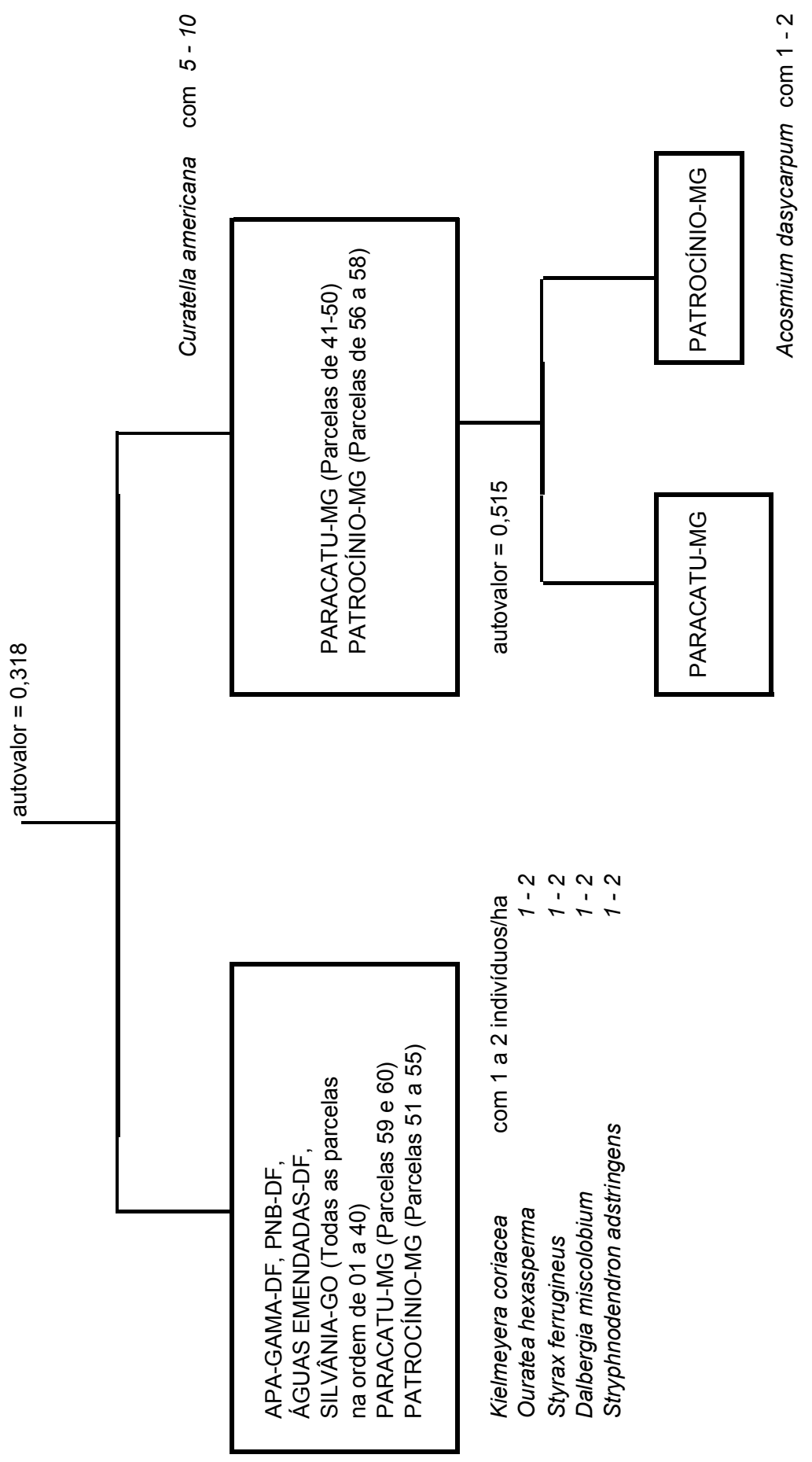

FIGURA 4 - Classificação pelo TWISPAN das 60 parcelas de cerrado na Chapada Pratinha, espécies indicadoras e nível de densidade. 
ção das diferenças nas densidades das populações.

A diversidade alfa foi maior no Distrito Federal-Silvânia enquanto a diversidade beta foi maior em Paracatu-Patrocínio. A maior diferenciação entre as parcelas em Paracatu-Patrocínio sugerem a necessidade de criação de unidades de conservação de grande porte nessa região, para abranger a variabilidade da flora ou de uma rede de unidades menores.

\section{Agradecimentos}

A equipe do projeto Biogeografia do Bioma Cerrado, ao FNMA pelo apoio financeiro e ao CNPq pela concessâo de bolsa. Este projeto foi parte do trabalho final de graduação da primeira autora que agradece aos membros da banca examinadora Professores Manoel Claúdio da Silva Júnior e Raimundo Paulo Barros Henriques pelas valiosas sugestões. Os autores agradecem também aos revisores do trabalho.

\section{Referências Bibliográficas}

Adámoli, J.; Macêdo, J.; Azevedo, L.G. \& Netto, J.M. 1987. Caracterização da região dos Cerrados. Pp. 33-98. In: Goedert, W.J. (ed.). Solos dos Cerrados: tecnologias e estratégias de manejo. Nobel, São Paulo.

Cochrane, T. T.; Sanchez, L.G.; Azevedo, L.G.; Porras, J. A. \& Garver, C.L. 1985. Land in tropical America. CIAT-EMBRAPA- CPAC, Cali. 3 vols.

Coutinho, L.M. 1978. O conceito de cerrado. Revista Brasileira de Botânica 1: 17-23.

Eiten, G. 1972. The cerrado vegetation of Brazil. Botanical Review 38: 201-341.

Eiten, G. 1984 .Vegetation of Brasília. Phytoecologia 12 : 271-292.

Felfili, J.M. \& Silva Júnior, M.C. 1992. Floristic composition, phytosociology and comparison of cerrado and gallery forests at Fazenda Água Limpa, Federal District, Brazil. Pp. 393-615. In: Furley, P.A., Proctor, J.A. and Ratter, J.A. (eds.) Nature and dynamics of forest-savanna boundaries. Chapman \& Hall, London.
Felfili J.M. \& Silva Junior. M. C. 1993. A comparative study of cerrado (sensu stricto) vegetation in Central Brazil. Journal of Tropical Ecology 9: 277-289.

Felfili, J.M.; Filgueiras, T.S.; Haridassan, M.; Silva Júnior, M.C.; Mendonça, R \& Rezende, A.V. 1994. Projeto biogeografia do bioma cerrado: Vegetação e solos. Caderno de Geociências do IBGE 12: $75-166$.

Felfili, J.M. 1995. Diversity, structure and dynamics of a gallery forest in central Brazil. Vegetatio 117:1-15.

Felfili, J. M.; Silva Junior, M. C.; Rezende, A . V.; Nogueira, P.E.; Walter, B. M. T., Silva, M. A . \& Encinas, J. I. 1997. Comparação Florística e Fitossociológica do Cerrado nas Chapadas Pratinha e dos Veadeiros. Pp. 6-11. In: Leite, L. \& Saito, C. H. (ed.). Contribuição ao conhecimento Ecológico do cerrado. Ed. Universidade de Brasília, Brasília.

Goodland, R. 1971. A physiognomic analysis of the "cerrado" vegetation of Central Brazil. Journal of Ecology 59: 411-419.

Goodland, R. \& Ferri, M. 1979. Ecologia do cerrado. USP e Itatiaia, Belo Horizonte. 193p.

Hill, M.O.; Bunce, R.G.H. \& Shaw, M.W. 1975. Indicator species analysis, a divisive polithetic method of classification, and its application to a survey of native pinewoods in Scotland. Journal of Ecology 63: 597-613.

Klink, C. A; Macedo, R. H. \& Muelller, C. C.1995. De grão em grão o cerrado perde espaço. Cerrado - Impactos do processo de ocupação. WWF/ PRÓ-CER, Brasília. 66 p.

Klink, C. A. 1996. Relação entre o desenvolvimento agrícola e a biodiversidade. Pp 25-27. In: Pereira, R. C. \& Nasser, L. C. B. (Ed.) Biodiversidade e produção sustentada de alimentos e fibras nos cerrados. $1^{\circ}$ Simpósio Internacional de savanas neotropicais. EMBRAPA, Brasília.

Magurran, A.E. 1988. Ecological diversity and its measurement. Chapman and Hall, London. 179p.

Mendonça, R. C.; Felfili, J.M.; Walter, B.M.T.; Silva Júnior, M.C.; Rezende, A.V.; Filgueiras, T.S. \& Nogueira, P.E. 1998. Flora vascular do Cerrado. Pp. 289-556. In: Sano, S. M. \& Almeida, S. P. Cerrado, Ambiente e Flora. EMBRAPA CPAC. Planaltina.

Mittermeyer, R.A., Myers, N.; Mittermeier, C.G. 1999. Hotspots Earth's biologically richest and most endagered terrestrial ecoregions. CEMEX Conservation International 
Oliveira Filho, A. T., Shepherd, G., Martins, F. R. \& Stubblebine, W. H. 1989. Enviromental factors affecting physiognomic floristic variation in an area of cerrado in Central Brazil. Journal of Tropical Ecology 5: 413-431.

Philip, M. S. 1994. Measuring tree and forests. Cambridge University Press, Cambridge. 310p.

Ratter, J. A.; Askew, G.P.; Montgomery, R.F. \& Gifford, D.R. 1978. Observations on forests of some mesotrophic soils in central Brazil. Revista Brasileira de Botânica 1:153-180.

Ratter, J. A. 1991. Guia para a vegetação da fazenda Água Limpa (Brasília-DF, Brasil). Editora Universidade de Brasília, Brasília. 138p.

Ratter, J. A. \& Dargie, T.C.D. 1992. An analysis of the floristic composition of 26 cerrado areas in Brazil. Edinburgh Journal of Botany, 49: 235250.

Ratter, J. A. et al. 1996. Analysis of the floristic composition of the Brazilian cerrado vegetation II: Comparison of the wood vegetation of 98 areas. Edinburgh, Journal of Botany 53 : 153-180.

Ribeiro, F.F.; Silva, J.V.S \& Azevedo, L. G. 1981. Estrutura composição florística em tipos fisionômicos dos cerrados e sua interação com alguns parâmetros do solo. Pp. 141-156. In: Anais do $32^{\circ}$ Congresso Nacional de Botânica. Sociedade Botânica do Brasil, Teresina.

Whittaker, R. H. 1960. Vegetation of the Siskioy Mountains, Oregon and California. Ecological

Monographs 30: 279-338. 